\title{
Knockdown of long non-coding RNA PEG10 inhibits growth, migration and invasion of gastric carcinoma cells by up-regulating miR-3200
}

\author{
J. WANG*, X. Q. CHÜ, D. ZHANG, D. F. KONG* \\ Department of Gastrointestinal Surgery, Jining No. 1 People’s Hospital, Jining 272000, Shandong, China \\ ${ }^{*}$ Correspondence: kongdongfang72@126.com

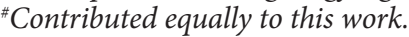

Received December 4, 2017 / Accepted March 21, 2018

\begin{abstract}
Gastric cancer is the major cause of cancer-related death worldwide. The aberrant expression of the PEG10 paternally expressed gene 10 is involved in the development of a range of cancers. Although the biological function and the underling mechanism of PEG10 in human gastric carcinoma are still unknown, LncRNA PEG10 knockdown could present a promising therapeutic strategy for the treatment of gastric cancer. Herein, the expression of PEG10, miR-3200 and AEG1 in human gastric carcinoma NCI-N87 cells was altered by cell transfection assay, and the cell viability, migration, invasion, and apoptosis were determined by trypan blue exclusion, Transwell assay and flow cytometry, respectively. Gene RNA and protein expression levels were analyzed by real-time PCR and Western blot and the Luciferase reporter assay determined miR-3200's target gene. JNK and Wnt signal pathway protein expressions were then tested by Western blot. While PEG10 up-regulation was evident in clinical samples its knockdown effectively inhibited gastric carcinoma cell viability, migration and invasion but promoted cell apoptosis. This tumor-suppressive effect by PEG10 knockdown can be provided by up-regulating miR-3200 in vitro and in vivo. AEG1 is a direct miR-3200 target gene and miR-3200 suppresses NCI-N87 cells by negative AEG1 regulation. Furthermore, miR-3200 up-regulation most likely blocks the JNK and Wnt pathways by down-regulating AEG1. Finally, PEG10 knockdown has a carcinostatic role by up-regulating miR-3200, and the JNK and Wnt pathway block during this process further regulates AEG1 in gastric carcinoma cells.
\end{abstract}

Key words: gastric carcinoma, PEG10, miR-3200, AEG1, JNK pathway, Wnt pathway

Gastric cancer is a major cause of cancer death, and is the most common type of gastrointestinal malignancy, especially in East Asia [1]. Unfortunately, it is usually diagnosed at a terminal stage accompanied by malignant multiplication and extensive invasion [2]. Although standard chemotherapy protocols have reduced the 5-year mortality rate, there is still urgent need to find new prognostic biomarkers and therapeutic targets for gastric cancer [2-4].

Long noncoding RNAs (LncRNAs) are a series of regulatory RNA molecules with greater than 200 nucleotide length. Abundant studies have demonstrated that lncRNAs are aberrantly expressed in different cancer types and act as oncogenes or suppressors in tumor progression $[5,6]$. lncRNAs including RP11-357H14.17 [7], GHET1 [8] and DANCR [9] have recently been identified as new modulators in the origin and development of gastric cancer, and can therefore be employed as effective therapeutic targets for this disease.
Herein, we studied the effect of the paternally expressed PEG10 lncRNA on gastric carcinoma. PEG10 has 763bp and is located on human chromosome 7 between the 94,285,681 and $94,298,949$ base sites $[10,11]$ and its aberrant expression has been closely associated with malignancies such as esophageal cancer [12], hypopharyngeal squamous cell carcinoma [10] and diffuse large B cell lymphoma [13]. While PEG10 acts as an oncogene in gastric cancer cells and its knockdown effectively inhibits cell proliferation, migration and invasion and promotes apoptosis, little is known about PEG10's molecular mechanisms and potentiality in human gastric carcinoma.

Some lncRNAs regulate microRNAs (miRNAs) [14] and their important functions in cancer processes $[15,16]$. For example, deregulated miR-3200 is a proven tumor-suppression marker in breast cancer [17]. A recent report has also shown that IncRNA CAT104 binds to miR-3200, and the miR-3200 influence in breast cancer patient survival may therefore be modulated by this interaction [18]. 
The roles of lncRNA PEG10 and miR-3200, and their regulatory relationships in gastric carcinoma are unclear so this inspired us to investigate if $\mathrm{miR}-3200$ could be regulated by IncRNA PEG10. We first studied the function of PEG10 in NCI-N87 gastric carcinoma cells and then investigated interactions between PEG10, miR-3200 and the Astrocyte Elevated Gene 1 (AEG1) in order to establish the underlying action mechanisms. These results will hopefully provide a new direction for gastric carcinoma treatment.

\section{Patients and methods}

Ethics statement. All animal experiments followed the 'Guide for the Care and Use of Laboratory Animals' published by the National Institutes of Health. The experiments were also approved by the Clinical Research Ethics Committee of Jining No. 1 People's Hospital and informed consent was obtained from all recruited patients or their guardians.

Patients and sample collection. Gastric cancer tissues and normal gastric tissue samples were collected from 20 patients who underwent curative surgery for gastric cancer at Jining No. 1 People's Hospital between April 2014 and October 2017, and the histopathology was diagnosed by three pathologists. The fresh tissues were immediately preserved in liquid nitrogen, and treatments were performed according to NCCN guidelines.

Cell culture. Human gastric carcinoma cell line NCI-N87 was purchased from American Type Culture Collection (ATCC; Manassas, VA, USA) and cultivated in Dulbecco's modified Eagle's medium (DMEM; Gibco, Carlsbad, CA, USA), supplemented with $10 \%(\mathrm{v} / \mathrm{v})$ fetal bovine serum (FBS; Gibco). Cells were plated in tissue culture dishes and pre-cultured at $37^{\circ} \mathrm{C}$ and $5 \% \mathrm{CO}_{2}$ for 2-4 days until confluence was reached

Cell transfection. (1) pEX-PEG10 analysis; short-hairpin RNA (shRNA) targeting human lncRNA PEG10 was ligated into the U6/GFP/Neo plasmid (GenePharma, Shanghai, China) to construct sh-PEG10, and the plasmid carrying a non-targeting sequence formed the negative control (shNC) for sh-PEG10. The full-length of PEG10 sequences was sub-cloned into the pEX-2 plasmid (GenePharma) to construct $\mathrm{pEX}-\mathrm{PEG} 10$ and the empty $\mathrm{pEX}-2$ plasmid was the negative control ( $\mathrm{pEX}-\mathrm{NC}$ ).

(2) miR-3200 analysis; miR-3200 mimic, miR-3200 inhibitor and the appropriate negative controls (mimic NC or inhibitor NC) were synthesized from Life Technologies Corporation (Carlsbad, CA, USA). NCI-N87 cells were plated $6 \times 10^{5}$ cells/well in 6-well plates and $50 \mathrm{nM}$ miR-3200 mimic/mimic $\mathrm{NC}$ or $150 \mathrm{nM}$ inhibitor/inhibitor NC was transfected into the NCI-N87 cells by lipofectamine 3000 reagent (Life Technologies Corporation). The sequences were as follows. miR-3200 mimic: sense, 5'-AAUCUGAGAAGGCGCACAAGGU-3'; anti-sense: 5'-CUUGUGCGCCUUCUCAGAUUUU-3'. miR-3200 mimic NC: sense, 5'-AGAGAGUGGAAUCUGCGCAAAC-3'; anti-sense: 5'-GUUUGCGCAGAUUCCACU-
CUCU-3'. miR-3200 inhibitor: 5'-ACCUUGUGCGCCUUCUCAGAUU-3'; miR-3200 inhibitor NC, 5'-GUGAUUCGCCUACCUCAGUUCU-3'.

(3) AEG1 analysis, the full-length AEG1 sequence was sub-cloned into the pEX-2 plasmid (GenePharma) to construct $\mathrm{pEX}-\mathrm{AEG} 1$. These recombined plasmids and empty pEX-2 were transfected into NCI-N87 cells by lipofectamine 3000 reagent according to the manufacturer's instructions. After selection with $0.5 \mathrm{mg} / \mathrm{ml} \mathrm{G} 418$ (Sigma-Aldrich, St Louis, MO, USA) for approximately 4 weeks, stably transfected NCI-N87 cells were successfully obtained.

Cell viability. Live cell numbers were determined in a blood cell counting chamber by trypan blue exclusion and cells were trypsinized and stained with trypan blue dye. The viable cells were counted daily for of 4 days and cell growth curves were plotted according to the viable cell numbers in each group.

Cell migration and invasion. NCI-N87 cell migration and invasion was assessed by Transwell chamber (Costar, Corning, NY, USA) with polycarbonic membrane $(6.5 \mathrm{~mm}$ diameter, $8 \mu \mathrm{m}$ pore size). For migration assay, the transfected cells were resuspended in $100 \mu \mathrm{l}$ serum-free medium at $5 \times 10^{5}$ cells $/ \mathrm{ml}$ density and added to the upper chamber and $600 \mu \mathrm{l}$ complete medium was added to the lower chamber. After incubation at $37^{\circ} \mathrm{C}$, non-migrated cells on the upper membrane surface were mechanically removed. Cells which migrated to the lower side of the membrane were stained with crystal violet for 15 minutes and counted under a microscope in five randomly selected fields. The invasion assay then followed the migration assay steps; the Transwell membrane was coated with $80 \mu \mathrm{l}$ of Matrigel solution ( $500 \mathrm{ng} / \mu \mathrm{l}$; BD, Franklin Lakes, NJ, USA) and incubated at $37^{\circ} \mathrm{C}$ for 4 hours.

Cell apoptosis. The NCI-N87 cells were seeded in a 6-well culture plate after transfection. Cells were collected 48 hours after incubation, washed with phosphate-buffered saline (PBS) and resuspended in $1 \times$ binding buffer at $1 \times 10^{6}$ cells $/ \mathrm{ml}$ density. $5 \mu \mathrm{l}$ Annexin V-FITC and $5 \mu \mathrm{l}$ PI were added to the cell suspension and cultured for 15 minutes in the dark at room temperature, and cell apoptosis was analyzed by flow cytometry (Becton Dickinson, San Jose, CA, USA).

Quantitative real-time PCR (qRT-PCR). Total RNA was extracted from cells and tissues by Trizol reagent (Life Technologies Corporation) according to the manufacturer's instructions. The One Step SYBR ${ }^{\circledast}$ PrimeScript ${ }^{\oplus}$ PLUS RT-RNA PCR Kit (TaKaRa Biotechnology, Dalian, China) was used for Real-Time PCR analysis to determine PEG10 expression level. The Taqman MicroRNA Reverse Transcription Kit and Taqman Universal Master Mix II with the TaqMan MicroRNA Assay of miR-3200 (Applied Biosystems, Foster City, CA, USA) then assessed miR-3200 expression levels. RNA PCR Kit (AMV) Ver.3.0 (TaKaRa Biotechnology) was used for AEG1 analysis. Expression data was normalized to the geometric mean of housekeeping gene GAPDH (for PEG10 and AEG1) and U6 (for miR-3200) to 
control the variability in expression levels and then analyzed by the $2^{-\Delta \Delta \mathrm{Ct}}$ method.

Primer sequences were synthesized by Invitrogen (Carlsbad, CA, USA). Primer sequence of lncRNA PEG10: forward, 5'-CATCCTTCCTGTCTTCGC-3'; reverse, 5'-CCCTCTTCCACTCCTTCTTT-3'. Primer sequence of GAPDH: forward, 5'-TGGTATCGTGGAAGGACTCA-3'; reverse, 5'-CCAGTAGAGGCAGGGATGA

T-3'. Primer sequence of AEG1: forward, 5' -TGTTGAAGTGGCTGAGGG-3'; reverse, 5'-CAGGAAATGATGCGGTTG-3'. Primer sequence of miR-3200: forward, 5'-GGG AATCTGAGAAGGCGCA-3'; reverse, 5'-CAGTGCGTGTCGTGGAGT-3'; Primer sequence of U6: forward, 5'-GCTTCGGCAGCACATATACTAAAAT-3'; reverse, 5'-CGCTTCACGAATTTGCGTGTCAT-3'.

Dual luciferase activity assay. The AEG1fragment containing the predicted miR-3200 binding site was amplified by PCR and cloned into a pmirGlO Dual-luciferase miRNA Target Expression Vector (Promega) to form the reporter vector AEG1-wild-type (AEG1-wt). To mutate the putative binding site of miR-3200 in the AEG1, the sequence of the putative binding site was replaced as indicated and is referred to as AEG1-mutated-type (AEG1-mt). The vectors and miR-3200 mimic were then co-transfected into cells by Lipofectamine 3000 (Life Technologies Corporation) and reporter assays were performed by the dual-luciferase assay system (Promega, Madison, WI, USA).
Western blot. RIPA lysis buffer (Beyotime Biotechnology, Shanghai, China) supplemented with phosphatase inhibitor cocktail (Pierce, Rockford, IL, USA) was used after transfection to extract protein from the NCI-N87 cells. Following quantification by the $\mathrm{BCA}^{\mathrm{Tm}}$ Protein Assay Kit (Pierce), equivalent proteins were separated by sodium dodecyl sulfate-polyacrylamide gel electrophoresis (SDS-PAGE) and the proteins in the gels were transferred to a polyvinylidene difluoride (PVDF) membrane. The membranes were blocked with non-fat milk at room temperature for 1 hour and then incubated at $4{ }^{\circ} \mathrm{C}$ overnight with primary antibodies against the following; Bcl-2 (ab32124), Bax (ab32503), pro caspase-3 (ab4051), cleaved caspase-3 (ab32042), caspase-9 (ab32539), cleaved caspase-9 (ab32539), Wnt3a (ab28472), Wnt5a (ab72583), $\beta$-catenin (ab32572), JNK (ab110724), p-JNK (ab47337), c-Jun (ab32137), p-c-Jun (ab32385), GAPDH (ab9485, Abcam, Cambridge, UK) and AEG1 (40-6500, Invitrogen).

The membranes were then washed and incubated with secondary antibody conjugated to horseradish peroxidase (HRP) for 1 hour at room temperature. Membranes were again rinsed and subjected to the Bio-Rad ChemiDoc ${ }^{\mathrm{Tm}}$ XRS system, and $200 \mu \mathrm{l}$ of Immobilon Western Chemiluminescent HRP Substrate (Millipore, Billerica, MA, USA) was added and signals were captured by Image Lab ${ }^{\mathrm{TN}}$ software (Bio-Rad, Shanghai, China).

Animal studies. Animal studies were conducted according to the 'National Institute of Health Animal Use
A

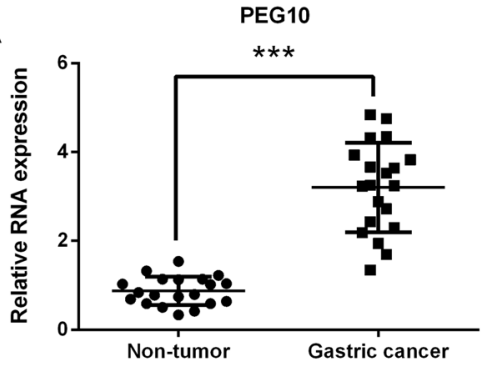

D

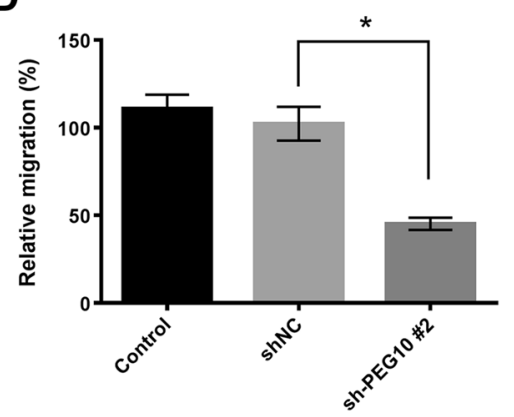

B

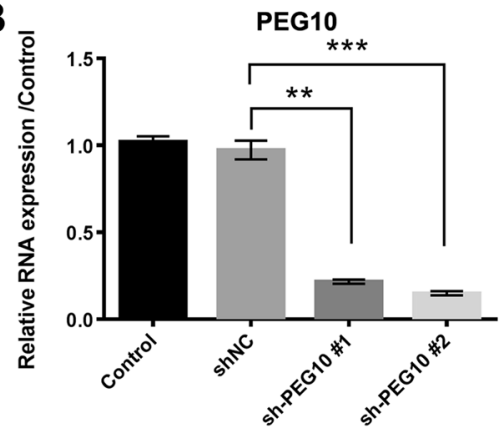

E

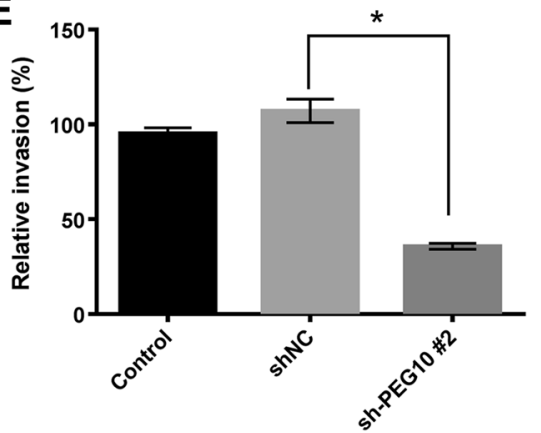

C

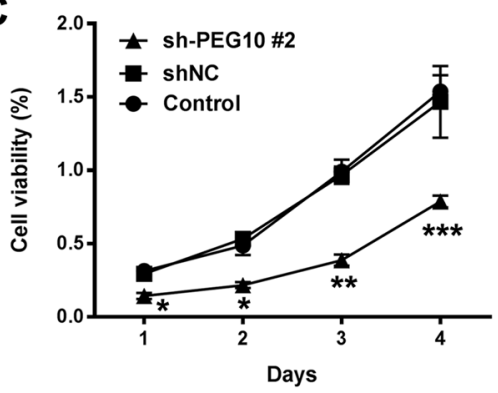

Figure 1. PEG10 was over-expressed in gastric cancer tissues, and knockdown of PEG10 inhibited NCI-N87 cell viability, migration and invasion. A) PEG10 was up-regulated in gastric cancer tissues. B) The RNA expression level of PEG10 after transfection with sh-PEG10 \#1 and \#2. C) The cell viability, D) cell migration, and E) cell invasion after down-regulating PEG10. Data is expressed as the mean $\pm \mathrm{SD} .{ }^{\star} \mathrm{p}<0.05,{ }^{\star *} \mathrm{p}<0.01,{ }^{\star * *} \mathrm{p}<0.001$. 

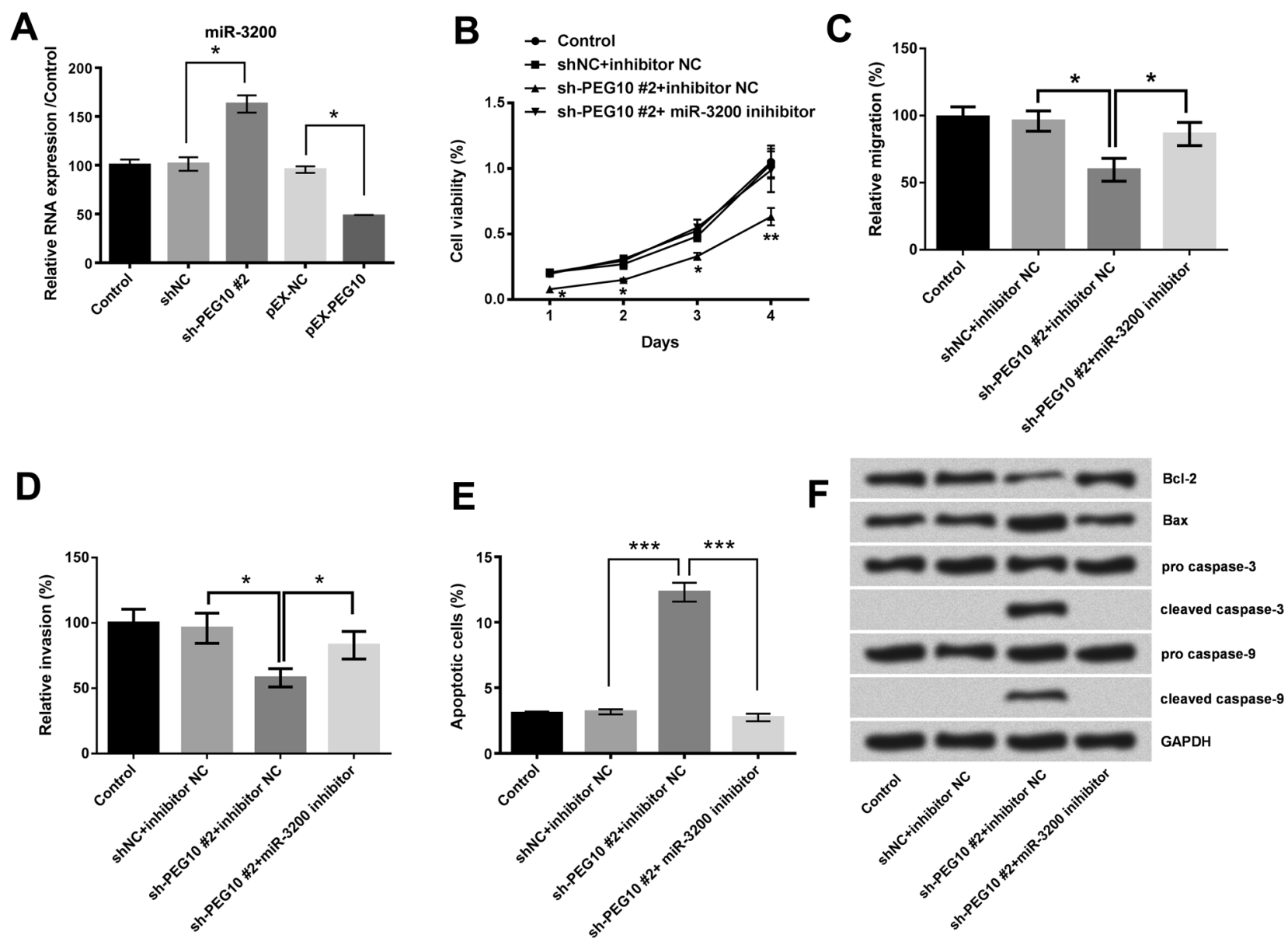

Figure 2. PEG10 suppressed NCI-N87 cells by miR-3200 regulation. A) Expression of miR-3200 after up- or down-regulating PEG10. B) The cell viability, C) cell migration, D) cell invasion, E) cell apoptotic rates, and F) apoptosis related proteins after NCI-N87 cells were transfected with sh-PEG10, miR-3200 inhibitor and their negative controls. Data is expressed as the mean $\pm \mathrm{SD} .{ }^{\star} \mathrm{p}<0.05,{ }^{* *} \mathrm{P}<0.01,{ }^{* * *} \mathrm{p}<0.001$.

Guidelines'. Approximately 4 week-old male nude mice were purchased from the Experimental Animals Center of Shandong province (Jinan, Shandong). $1 \times 10^{6} \mathrm{NCI}-\mathrm{N} 87$ cells from each group, transfected with shNC+inhibitor NC, sh-PEG10\#2+inhibitor NC, or sh-PEG10\#2+miR-3200 inhibitor, were injected into the animals' bilateral axilla. The tumors were harvested 4 weeks later and were weighed and the maximum diameter measured. This work was based on a blind randomized animal study protocol, so that mice were randomly separated into groups and 6 mice were eventually used for each group.

Statistical analysis. The data was expressed as the mean \pm SD from at least three separate experiments. Statistical analysis was conducted by Graphpad 6.0 statistical software (Graph Pad Software Inc, San Diego, CA, USA), and P values were calculated by one-way analysis of variance (ANOVA). P-values less than 0.05 were considered statistically significant.

\section{Results}

PEG10 knockdown inhibited viability, migration, and invasion of NCI-N87 cells. PEG10 expression in 20 freshly frozen gastric cancer samples was shown to be significantly up-regulated relative to non-tumor samples $(p<0.001$, Figure 1A). PEG10 was down-regulated by shRNA transfection and its effects on viability, migration, and invasion of gastric carcinoma NCI-N87 cells were as follows; sh-PEG10 effectively inhibited PEG10 expression in NCI-N87 cells compared to the shNC group and lower PEG10 expression was detected after transfection with sh-PEG10 \#2 ( $<<0.001)$ compared to sh-PEG10 \#1 ( $<<0.01$, Figure 1B).

Then sh-PEG10 \#2 was used for subsequent assays. NCI-N87 cell viability was detected daily for 4 days by trypan blue exclusion, and cell viability was obviously reduced after PEG10 silence (Figure 1C). Cell migration and invasion were then analyzed by Transwell assay, and both were signifi- 
cantly decreased compared to the shNC group (both $\mathrm{p}<0.05$, Figures $1 \mathrm{D}$ and E, Supplementary Figure 1). These results illustrated that PEG10 knockdown inhibited NCI-N87 cell viability, migration and invasion.

PEG10 silence suppressed growth, migration and invasion of NCI-N87 cells via up-regulation of miR-3200. qRT-PCR analysis showed that PEG10 knockdown facilitated miR-3200 expression and that PEG10 over-expression inhibited miR-3200 expression in NCI-N87 cells (both $\mathrm{p}<0.05$, Figure $2 \mathrm{~A}$ ). miR-3200 can be regulated by PEG10, so we expected miR-3200 to participate in NCI-N87 cell PEG10 function. To determine miR-3200's role, its expression was altered by cell transfection, with resultant over-expression in mimic-transfected cells and down-regulation in inhibitortransfected cells (Supplementary Figure 2). sh-PEG10 and miR-3200 inhibitor were then co-transfected into NCI-N87 cells to determine if miR-3200 was implicated in PEG10silencing tumor-suppression The inhibitory effect of PEG10 silencing on cell viability, migration and invasion were all abolished by miR-3200 inhibition (Figures 2B, C, and D, Supplementary Figure 3).

Cell apoptosis and related proteins were analyzed by flow cytometry and Western blot. Results determined that downregulating PEG10 dramatically increased apoptotic cell rate (Figure 2E), enhanced pro-apoptosis protein levels, including Bax, cleaved caspase-3/9 and decreased Bcl-2 anti-apoptotic protein level (Figure 2F). In contrast, miR-3200 inhibition abolished the apoptosis-promoting effect of PEG10 knockdown. In conclusion, the combined data suggests that silencing PEG10 inhibits gastric carcinoma cell growth, migration and invasion by up-regulating miR-3200.

AEG1 was a target of miR-3200. The AEG1mRNA level was significantly down-regulated by the miR-3200 mimic $(\mathrm{p}<0.05)$, but largely up-regulated by the inhibitor $(\mathrm{p}<0.01$, Figure $3 \mathrm{~A}$ ). The AEG1 protein expression level was then determined and this produced the similar results displayed in Figure 3B. The AEG1 expression was negatively regulated by miR-3200 both in mRNA level and protein level, so we expected AEG1 to have relevant miR-3200 binding sites. This was confirmed by dual-luciferase reporter assay. Co-transfection of miR-3200 mimic and AEG1-wt strongly decreased luciferase activity, while co-transfection of miR-3200 mimic and AEG1-mt did not change it (Figure 3C). The combined results enable us to conclude that AEG1 is the direct miR-3200 target of miR-3200 and that it is negatively regulated by miR-3200 in NCI-N87 cells.

miR-3200 suppressed growth, migration and invasion of NCI-N87 cells by negative regulation of AEG1. Herein, we up-regulated AEG1 by transfecting pEX-AEG1 into NCI-N87 cells because miR-3200 regulates AEG1, and AEG1 could well be implicated in miR-3200 action. Both qRT-PCR and Western blot determined AEG1 expression was up-regulated (Supplementary Figure 4). We established that AEG1 expression in both mRNA and protein levels were down-regulated when cells were transfected with miR-3200

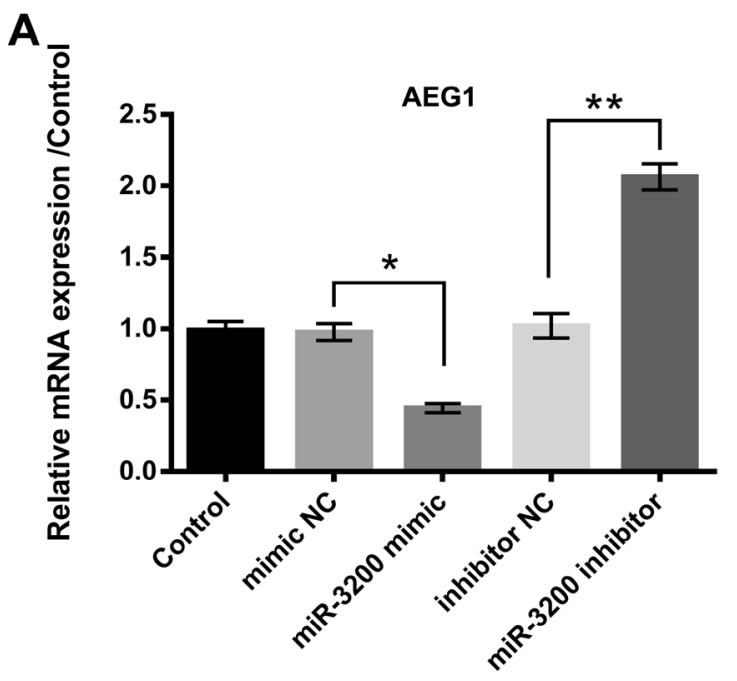

B

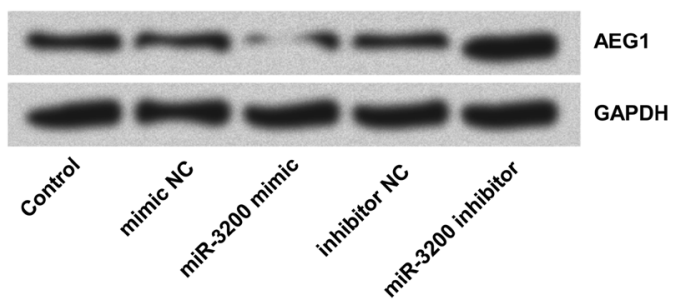

C

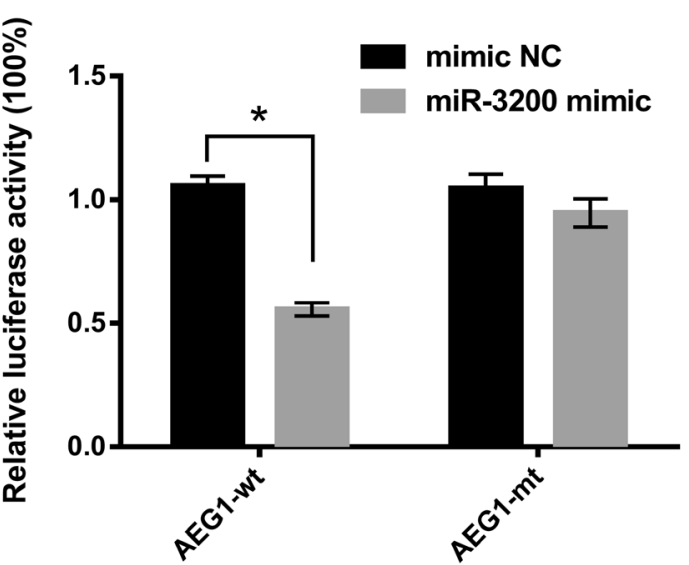

Figure 3. AEG1 is a direct miR-3200 target. A) The mRNA and B) protein level expressions of AEG1 after up- or down-regulating miR-3200. C) Luciferase activity in NCI-N87 cells co-transfected with miR-3200 mimic and luciferase reporters containing AEG1-wt or AEG1-mt transcript. Data is expressed as mean $\pm \mathrm{SD} .{ }^{\star} \mathrm{p}<0.05,{ }^{\star *} \mathrm{p}<0.01$.

mimic and that both levels were recovered and even enhanced when cells were co-transfected with miR-3200 mimic and pEX-AEG1 (Figures 4A and B). In addition, although miR-3200 over-expression effectively inhibited cell viability (Figure 4C), migration (Figure 4D, Supplementary Figure 5) and invasion (Figure 4E, Supplementary Figure 5), it promoted cell apoptosis (Figures $4 \mathrm{~F}$ and $\mathrm{G}$ ) and thus revealed its antagonism to gastric carcinoma. 

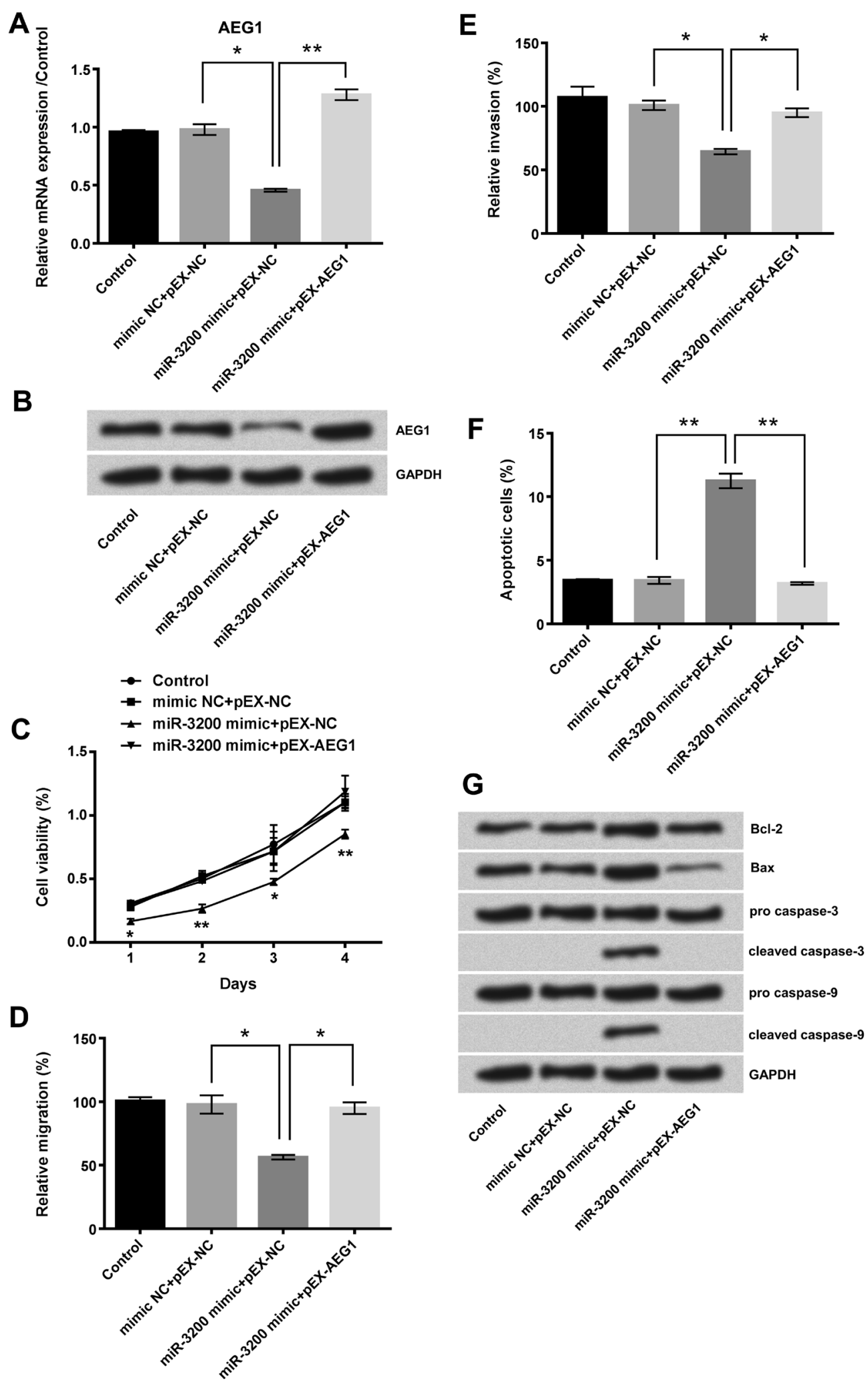

Figure 4. miR-3200 suppressed NCI-N87 cells via negatively regulating AEG1. A) AEG1 mRNA level, B) AEG1 protein level, C) cell viability, D) cell migration, E) cell invasion, F) cell apoptosis, and G) cell apoptosis related proteins after NCI-N87 cells were transfected with miR-3200 mimic, pEXAEG1 and their negative controls. Data is expressed as mean $\pm \mathrm{SD} .{ }^{*} \mathrm{p}<0.05,{ }^{* *} \mathrm{p}<0.01$. 
However, co-transfection of miR-3200 mimic and pEX-AEG1 rescued the tumor-inhibiting effect of miR-3200 over-expression on NCI-N87 cells, and the combined results suggest that AEG1 is an miR-3200 target and that it is involved in miR-3200-induced alteration of cell viability, migration, invasion and apoptosis.

miR-3200 inhibited JNK and Wnt pathways in NCI-N87 cells by negative regulation of AEG1. We analyzed the respective effects of miR-3200 and AEG1 on activation of the JNK and Wnt pathways. The Western blot results revealed the following effects on the key pathway components; (1) expression of p-JNK and p-c-Jun were inhibited in miR-3200-overexpressed cells but enhanced in AEG1-over-expressed cells (Figure 5A); (2) expressions of Wnt3a, Wnt5a and $\beta$-catenin were decreased in miR-3200-over-expressed cells and increased in AEG1-over-expressed cells (Figure 5B) and (3). AEG1 over-expression activated both JNK and Wnt pathways.
Our following experiment examined if miR-3200 was implicated in the AEG1 regulatory effect on JNK and Wnt pathway activation in the NCI-N87 cells. The results showed that p-JNK, p-c-Jun, Wnt3a, Wnt5a and $\beta$-catenin expression in the miR-3200 mimic+pEX-AEG1 treatment group were all significantly inhibited compared to the pEX-AEG1 treatment group. This therefore indicated that miR-3200 could inactivate the JNK and Wnt pathways by targeting AEG1 in the NCI-N87 cells.

Suppression of PEG10 inhibited tumor growth in vivo by up-regulating miR-3200. Investigation if lncRNA PEG10 mediated tumorigenesis in vivo established that although tumor xenograft volume and weight were suppressed in the sh-PEG10 group compared to the control group, both were increased after miR-3200 was silenced (Figures 6A B). This suggests that down-regulating PEG10 inhibited tumor growth in vivo by up-regulating miR-3200.
A
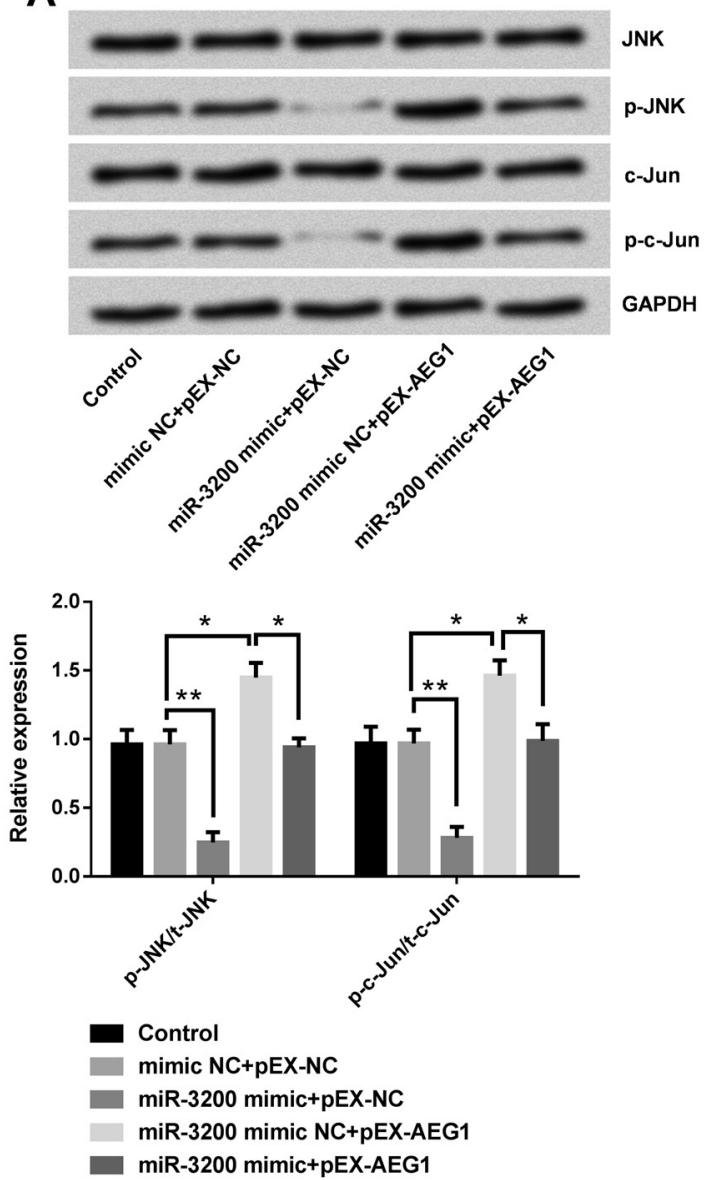

B
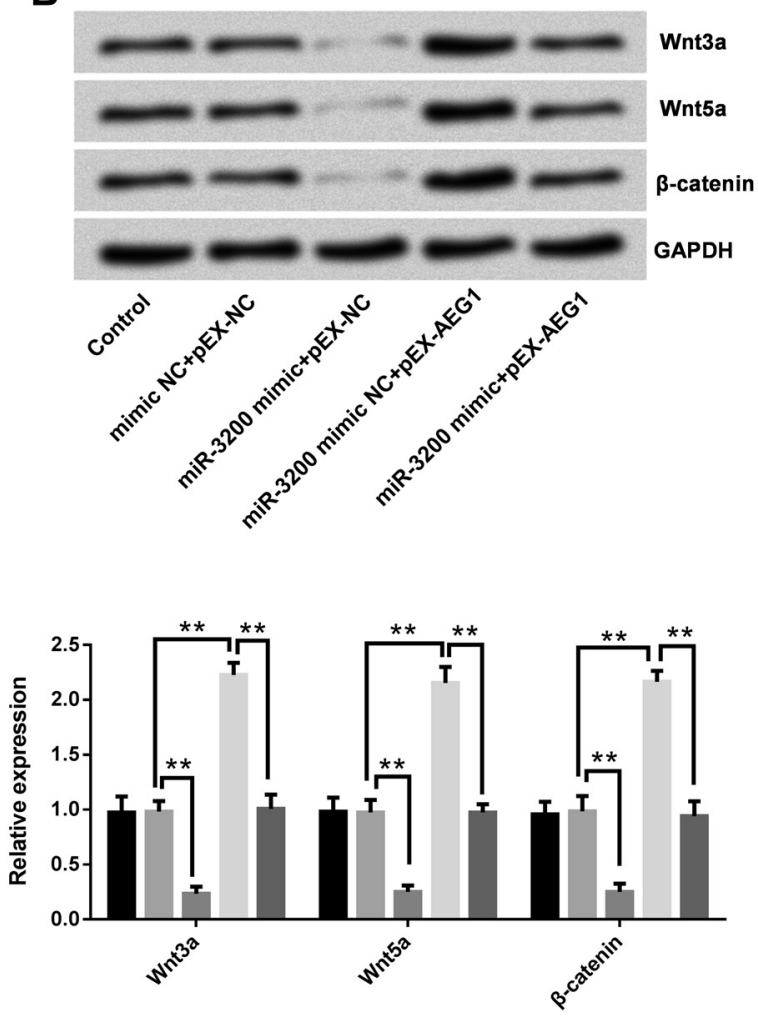

Control

mimic NC+pEX-NC

miR-3200 mimic+pEX-NC

miR-3200 mimic NC+pEX-AEG1

miR-3200 mimic+pEX-AEG1

Figure 5. JNK and Wnt pathway activation is affected by AEG1. Protein expression of key components of A) the JNK and B) Wnt signaling pathways after NCI-N87 cells were transfected with mimic NC, pEX-NC, miR-3200 mimic or/and pEX-AEG1. 

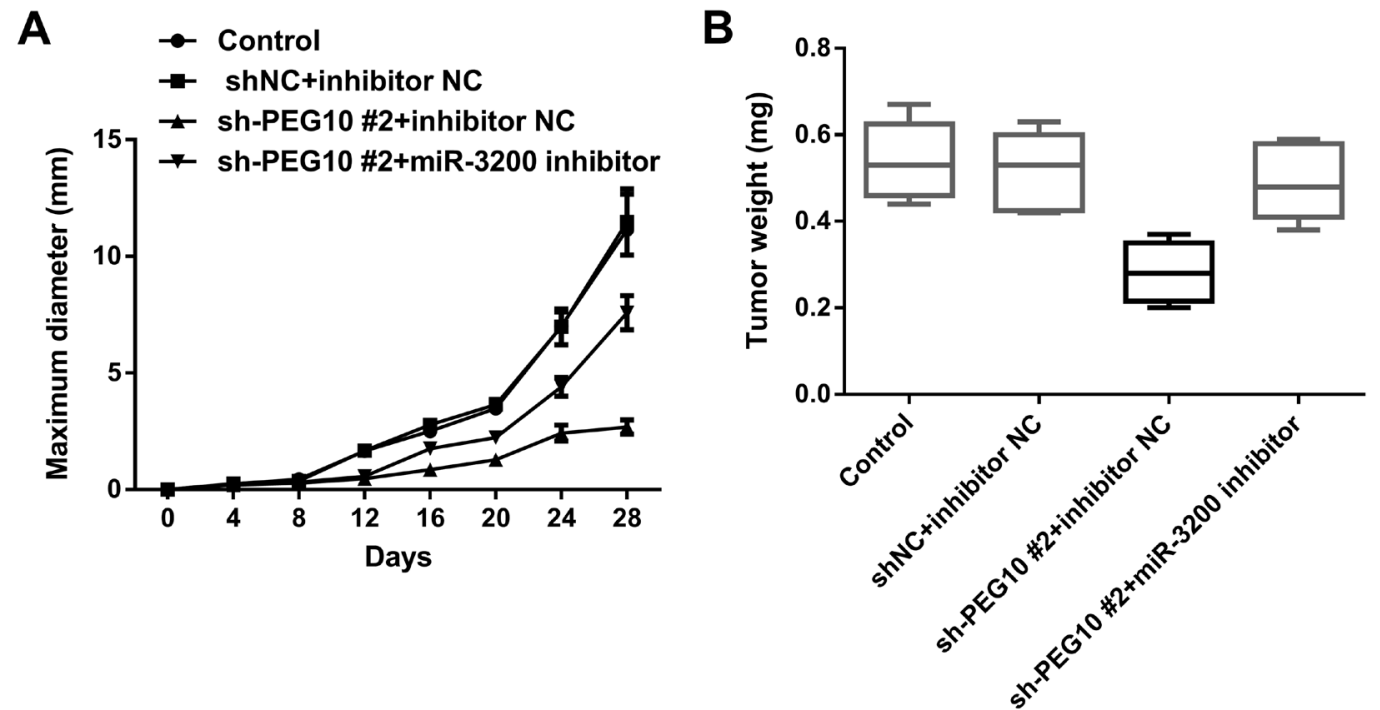

Figure 6. Down-regulating PEG10 inhibited the growth of transplanted tumor by up-regulating miR-3200. A) The maximum diameter and B) tumor weight were detected after PEG10 down-regulation or/and miR-3200 down-regulation.

\section{Discussion}

Gastric carcinoma poses a great threat to global public health because of its high incidence and mortality rates, and increasing evidence has revealed lncRNA involvement in tumor initiation and progression [19]. Therefore, it is important to identify gastric cancer-related lncRNAs and investigate their specific roles in gastric carcinogenesis [20].

Research has established that LncRNA PEG10 is overexpressed in diffuse large B cell lymphoma, esophageal cancer, and hypopharyngeal squamous cell carcinoma cell lines and tumor tissues compared to their normal counterparts $[10,12,13]$; thus defining PEG10 as an oncogenic lncRNA. Further confirmation includes; (1) Zhao et al. reported that PEG10 over-expression accelerates tumorigenic proliferation, invasion and migration [10]: (2) Zang et al. demonstrated that PEG10 down-regulation inhibited proliferation and invasion and promoted apoptosis in the EC9706 and KYSE150 esophageal cancer cell lines [12] and (3) Ishii et al reported that elevated PEG10 expression was found in gastric cancer tissues. This research supports our result that PEG10 was up-regulated in gastric cancer patients and that silencing PEG10 inhibited gastric carcinoma cell viability, migration and invasion and promoted NCI-N87 cell apoptosis.

Since the precise mechanism of PEG10 mediation of cancer cellular processes remains unresolved, this contribution focused on the possible action mechanism of PEG10 in gastric carcinoma cells. We found that silencing PEG10 suppressed NCI-N87 cells by up-regulating miR-3200. Moreover, this is not the first finding that miR-3200 is regulated by LncRNA because Guo et al reported negative correlation between LncRNA CAT104 and miR-3200 in breast cancer, and their sequence alignment showed that CAT104 could bind to miR-3200 [18]. Elevated levels of miR-3200 have also contributed to better pathological response and lower risk of breast cancer relapse [17].

However, very little has previously been documented on the role of miR-3200 in gastric cancer; especially in regard to functions and action mechanisms. Herein, AEG1 was established as a direct miR-3200 target, and miR-3200 most likely influenced NCI-N87 cell viability, apoptosis, migration and invasion by regulating AEG1 expression. Further, AEG1 is widely reported to regulate development and progression in various cancers; including gastric cancer [21, 22]. Inhibiting AEG1 expression clearly inhibited NCI-N87 cell growth and enhanced cell apoptosis [21].

In addition, Guo et al. also reported that miR- 137 functions as a tumor suppressor by inhibiting AEG1 [23], and this supports our research that miR-3200 has a tumor-suppressive role by inhibiting AEG1. Specifically, the over-expression of miR-3200 in NCI-N87 cells decreased cell viability, migration, and invasion and also induced apoptosis, while restoring AEG1 significantly reduced the miR-3200-induced inhibitory effects on cell growth, migration, and invasion.

JNK is a highly complex signaling pathway involved in proliferation, differentiation and apoptosis of gastric cancer cells, and also in their progression, migration and invasion [24-26]. Herein, we established that the JNK pathway was inhibited by miR-3200 by down-regulating AEG1 and this JNK pathway regulation by AEG1 is supported by other studies $[27,28]$. The Wnt signaling pathway is one of the main intracellular signal transduction pathways [29] and it is closely associated with development in a variety of human cancers. It has an important role in estimating biological 
factors in gastric cancer [30], and we determined herein that this pathway is regulated by AEG1. Similarly, AEG1 participated in this cancer by mediating the $\mathrm{Wnt} / \beta$-catenin pathway $[21,29,31]$.

In conclusion, our combined results established that silencing PEG10 inhibited the viability, migration and invasion of NCI-N87 cells and promoted their apoptosis by up-regulating miR-3200. Moreover, miR-3200 over-expression inhibited AEG1 expression and blocked JNK and Wnt pathway activity, thus further suppressing gastric cancer cell malignancy. Finally, we present the PEG10-miR-3200-AEG1 axis as a promising strategy for gastric cancer therapy.

\section{References}

[1] HERSZENYI L, TULASSAY Z. Epidemiology of gastrointestinal and liver tumors. Eur Rev Med Pharmacol Sci 2010; 14: 249-258.

[2] SIEGEL R, NAISHADHAM D, JEMAL A. Cancer statistics, 2013. CA Cancer J Clin 2013; 63: 11-30. https://doi. org/10.3322/caac. 21166

[3] HARTGRINK HH, JANSEN EP, VAN GRIEKEN NC, VAN DE VELDE CJ. Gastric cancer. Lancet 2009; 374: 477-490. https://doi.org/10.1016/S0140-6736(09)60617-6

[4] ZHUANG M, GAO W, XU J, WANG P, SHU Y. The long non-coding RNA H19-derived miR-675 modulates human gastric cancer cell proliferation by targeting tumor suppressor RUNX1. Biochem Biophys Res Commun 2014; 448: 315322. https://doi.org/10.1016/j.bbrc.2013.12.126

[5] WAPINSKI O, CHANG HY. Long noncoding RNAs and human disease. Trends Cell Biol 2011; 21: 354-361. https://doi. org/10.1016/j.tcb.2011.04.001

[6] SILVA JM, PEREZ DS, PRITCHETT JR, HALLING ML, TANG $\mathrm{H}$ et al. Identification of Long stress-induced noncoding transcripts that have altered expression in cancer. Genomics 2010; 95: 355-362. https://doi.org/10.1016/j.ygeno.2010.02.009

[7] YANG B, LUO T, ZHANG M, LU Z, XUE X et al. The novel long noncoding RNA RP11-357H14.17 acts as an oncogene by promoting cell proliferation and invasion in diffuse-type gastric cancer. Onco Targets Ther 2017; 10: 2635-2643. https://doi.org/10.2147/OTT.S134121

[8] ZHANG X, BO P, LIU L, ZHANG X, LI J. Overexpression of long non-coding RNA GHET1 promotes the development of multidrug resistance in gastric cancer cells. Biomed Pharmacother 2017; 92: 580-585. https://doi.org/10.1016/j. biopha.2017.04.111

[9] HAO YP, QIU JH, ZHANG DB, YU CG. Long noncoding RNA DANCR, a prognostic indicator, promotes cell growth and tumorigenicity in gastric cancer. Tumour Biol 2017; 39: 1010428317699798. https://doi. org/10.1177/1010428317699798

[10] ZHAO M, SUN D, LI X, XU Y, ZHANG H et al. Overexpression of long noncoding RNA PEG10 promotes proliferation, invasion and metastasis of hypopharyngeal squamous cell carcinoma. Oncol Lett 2017; 14: 2919-2925. https://doi. org/10.3892/ol.2017.6498
[11] ONO R, KOBAYASHI S, WAGATSUMA H, AISAKA K, KOHDA $\mathrm{T}$ et al. A retrotransposon-derived gene, PEG10, is a novel imprinted gene located on human chromosome 7q21. Genomics 2001; 73: 232-237. https://doi.org/10.1006/ geno.2001.6494

[12] ZANG W, WANG T, HUANG J, LI M, WANG Y et al. Long noncoding RNA PEG10 regulates proliferation and invasion of esophageal cancer cells. Cancer Gene Ther 2015; 22: 138144. https://doi.org/10.1038/cgt.2014.77

[13] PENG W, FAN H, WU G, WU J, FENG J. Upregulation of long noncoding RNA PEG10 associates with poor prognosis in diffuse large B cell lymphoma with facilitating tumorigenicity. Clin Exp Med 2016; 16: 177-182. https://doi. org/10.1007/s10238-015-0350-9

[14] YU F, LU Z, CAI J, HUANG K, CHEN B et al. MALAT1 functions as a competing endogenous RNA to mediate Rac1 expression by sequestering miR-101b in liver fibrosis. Cell Cycle 2015; 14: 3885-3896. https://doi.org/10.1080/1538410 1.2015.1120917

[15] ZHANG Y, DUTTA A, ABOUNADER R. The role of microRNAs in glioma initiation and progression. Front Biosci (Landmark Ed) 2012; 17: 700-712.

[16] IORIO MV, CROCE CM. MicroRNA dysregulation in cancer: diagnostics, monitoring and therapeutics. A comprehensive review. EMBO Mol Med 2012; 4: 143-159. https:// doi.org/10.1002/emmm.201100209

[17] AL-KHANBASHI M, CARAMUTA S, ALAJMI AM, ALHADDABI I, AL-RIYAMI $M$ et al. Tissue and Serum miRNA Profile in Locally Advanced Breast Cancer (LABC) in Response to Neo-Adjuvant Chemotherapy (NAC) Treatment. PLoS One 2016; 11: e0152032. https://doi.org/10.1371/ journal.pone.0152032

[18] GUO W, WANG Q, ZHAN Y, CHEN X, YU Q et al. Transcriptome sequencing uncovers a three-long noncoding RNA signature in predicting breast cancer survival. Sci Rep 2016; 6: 27931. https://doi.org/10.1038/srep27931

[19] BOLHA L, RAVNIK-GLAVAC M, GLAVAC D. Long Noncoding RNAs as Biomarkers in Cancer Dis Markers 2017; 2017: 7243968. https://doi.org/10.1155/2017/7243968

[20] XIE M, NIE FQ, SUN M, XIA R, LIU YW et al. Decreased long noncoding RNA SPRY4-IT1 contributing to gastric cancer cell metastasis partly via affecting epithelial-mesenchymal transition. J Transl Med 2015; 13: 250. https://doi. org/10.1186/s12967-015-0595-9

[21] JIAN-BO X, HUI W, YU-LONG H, CHANG-HUA Z, LONG-JUAN Z et al. Astrocyte-elevated gene-1 overexpression is associated with poor prognosis in gastric cancer. Med Oncol 2011; 28: 455-462. https://doi.org/10.1007/s12032010-9475-6

[22] LI G, WANG Z, YE J, ZHANG X, WU H et al. Uncontrolled Inflammation Induced by AEG-1 Promotes Gastric Cancer and Poor Prognosis. Cancer Res 2014; 74: 5541-5552. https://doi.org/10.1158/0008-5472.CAN-14-0968

[23] GUO J, XIA B, MENG F, LOU G. miR-137 suppresses cell growth in ovarian cancer by targeting AEG-1. Biochem Biophys Res Commun 2013; 441: 357-363. https://doi. org/10.1016/j.bbrc.2013.10.052 
[24] FU H, HU Z, WEN J, WANG K, LIU Y. TGF- $\beta$ promotes invasion and metastasis of gastric cancer cells by increasing fascin 1 expression via ERK and JNK signal pathways. Acta Biochim Biophys Sin (Shanghai) 2009; 41: 648-656.

[25] LIN HH, CHEN JH, KUO WH, WANG CJ. Chemopreventive properties of Hibiscus sabdariffa L. on human gastric carcinoma cells through apoptosis induction and JNK/p38 MAPK signaling activation. Chem Biol Interact 2007; 165: 59-75. https://doi.org/10.1016/j.cbi.2006.10.011

[26] SHIBATA W, MAEDA S, HIKIBA Y, YANAI A, SAKAMOTO $\mathrm{K}$ et al. c-Jun NH2-terminal kinase 1 is a critical regulator for the development of gastric cancer in mice. Cancer Res 2008; 68: 5031-5039. https://doi.org/10.1158/0008-5472. CAN-07-6332

[27] CHANG Y, LI B, XU X, SHEN L, BAI H et al. LentivirusMediated Knockdown of Astrocyte Elevated Gene-1 Inhibits Growth and Induces Apoptosis through MAPK Pathways in Human Retinoblastoma Cells. PLoS One 2016; 11: e0148763. https://doi.org/10.1371/journal.pone.0148763
[28] WANG F, KE ZF, WANG R, WANG YF, HUANG LL et al. Astrocyte elevated gene-1 (AEG-1) promotes osteosarcoma cell invasion through the JNK/c-Jun/MMP-2 pathway. Biochem Biophys Res Commun 2014 ;452: 933-939. https://doi. org/10.1016/j.bbrc.2014.09.009

[29] LI M, DAI Y, WANG L, LI L. Astrocyte elevated gene-1 promotes the proliferation and invasion of breast cancer cells by activating the Wnt/beta-catenin signaling pathway. Oncol Lett 2017; 13: 2385-2390. https://doi.org/10.3892/ ol.2017.5695

[30] ZHANG H, XUE Y. Wnt pathway is involved in advanced gastric carcinoma. Hepatogastroenterology 2008; 55: 11261130.

[31] HE W, HE S, WANG Z, SHEN H, FANG W et al. Astrocyte elevated gene-1(AEG-1) induces epithelial-mesenchymal transition in lung cancer through activating Wnt/betacatenin signaling. BMC Cancer 2015; 15: 107. https://doi. org/10.1186/s12885-015-1124-1 